Horizons philosophiques

\title{
Heidegger, le philosophe et la cité
}

\section{Sur la filliation philosophique d'un engagement politique}

\section{Martine Béland}

Volume 16, numéro 2, printemps 2006

Héritage et réception de la pensée existentialiste

URI : https://id.erudit.org/iderudit/801321ar

DOI : https://doi.org/10.7202/801321ar

Aller au sommaire du numéro

Éditeur(s)

Collège Édouard-Montpetit

ISSN

1181-9227 (imprimé)

1920-2954 (numérique)

Découvrir la revue

\section{Citer cet article}

Béland, M. (2006). Heidegger, le philosophe et la cité : sur la filiation philosophique d'un engagement politique. Horizons philosophiques, 16(2), 97-118. https://doi.org/10.7202/801321ar d'utilisation que vous pouvez consulter en ligne.

https://apropos.erudit.org/fr/usagers/politique-dutilisation/ 


\section{Heidegger, le philosophe et la cité Sur la filiation philosophique d'un engagement politique ${ }^{1}$}

Sans conteste, l'engagement politique est une dimension importante de la philosophie de l'existence au Xxe siècle. Qu'il s'agisse de l'entreprise des Temps modernes lancée par Sartre et Merleau-Ponty, de la morale sartrienne qui s'élève contre toute forme de domination, des écrits féministes de Simone de Beauvoir ou de l'engagement d'Albert Camus, la voix politique semble toute naturelle pour la philosophie de l'existence. Toutefois, les voies politiques qu'elle a suivies ne sont pas uniformes, au point qu'il vaudrait mieux parler au pluriel des philosophies de l'existence. Martin Heidegger n'est pas à proprement parler un existentialiste, et il a lui-même cherché à se distinguer de cette appellation en 1946 dans sa Lettre sur l'humanisme qui se présente comme une répartie lancée à la conférence de Sartre L'existentialisme est un humanisme. Quel que soit le terme précis qu'il faille employer pour nommer la pensée heideggerienne, il demeure que Heidegger a élaboré, principalement dans la première moitié de sa carrière, une philosophie de l'existence humaine. Or, cette pensée a trouvé une voie d'engagement politique pour le moins problématique : en acceptant en 1933 le poste de recteur de l'université de Fribourg, le philosophe a cautionné le national-socialisme. Avec Heidegger, une philosophie de l'existence s'engage politiquement pour un régime d'extrême-droite : voilà certes un cas qui mérite examen?2.

En fonction de l'engagement politique de 1933-1934, de la rhétorique par laquelle Heidegger l'a affirmé, et de la position politique qui s'est par après dessinée en filigrane dans les écrits du philosophe, de nombreux commentateurs sont d'avis que l'œuvre de Heidegger est à condamner (une position qu'ont par exemple défendue Luc Ferry, Alain Renaut et Arno Münster ${ }^{3}$ ), voire même à retirer des rayons de philosophie pour plutôt trouver place sur les rayons d'histoire (une position affirmée dans le récent livre d'Emmanuel Faye ${ }^{4}$ ). La réflexion que nous développons en ces pages n'entend pas suivre cette voie maintes fois empruntée, mais plutôt s'inspirer de celle tracée par un Nicolas Tertulian ou un Jean-Michel Palmier ${ }^{5}$ qui cherchent à rendre compte de la dimension sémantique de l'œuvre de 
Heidegger en la resituant dans le champ qui l'a vue naître. Si des chercheurs comme Domenico Losurdo et Pierre Bourdieu ont habilement travaillé à cerner les causes sociologiques de l'engagement politique de Heideggerb, nous cherchons pour notre part à mettre au jour ses causes proprement philosophiques. C'est la filiation philosophique de la dimension politique de la pensée heideggerienne qui nous intéresse. Une telle analyse ne signe pas le rejet de la pensée de Heidegger : elle souhaite plutôt situer les limites à l'intérieur desquelles cette philosophie peut aider à penser la modernité politique.

Dans les pages qui suivent, nous nous pencherons plus précisément sur l'engagement politique de Heidegger de manière à dégager ce qu'il suggère quant aux liens de la philosophie au politique, ou du philosophe à la cité. En effet, nous croyons que l'engagement politique heideggerien offre à la philosophie l'occasion d'une réflexion sur le rapport parfois ambigu, et souvent problématique, qu'elle entretient avec le politique. Notre travail sera mené en trois temps. Nous nous intéresserons d'abord directement à l'engagement politique de Heidegger, en proposant une analyse de son Discours de rectorat (1933). Cela nous permettra de nous pencher en deuxième temps sur le rapport du philosophe au politique tel que l'engagement politique de Heidegger permet de le penser. Nous devrons d'abord examiner le retrait du philosophe par rapport au politique suite à l'échec de son essai d'engagement, et souligner ensuite la teneur générale des allusions politiques qui se sont dessinées en filigrane dans les textes du philosophe jusqu'aux années 1960. Cette analyse du rapport du philosophe Heidegger à la cité nous permettra en un troisième temps d'en retracer la filiation philosophique. Nous proposerons que les sources philosophiques du rapport heideggerien du philosophe au politique sont d'une part chez Ernst Jünger, et d'autre part chez Friedrich Nietzsche. Avec cette étude de la filiation philosophique du rapport du philosophe au politique chez Heidegger, bien qu'elle soit limitée dans son objectif précis, nous espérons participer à la vaste entreprise, peut-être à jamais en chantier, d'une réflexion sur le rapport du philosophe à la cité.

\section{Un engagement politique}

C'est le 27 mai 1933 que Heidegger prononce son Discours de rectorat à l'université de Fribourg, sous le titre Die Selbstbehauptung der deutschen Universität ${ }^{7}$. Le philosophe nouvellement recteur y 
livre une exhortation aux étudiants et aux professeurs afin de les mobiliser en temps de crise politique. Si l'université a un rôle capital à jouer dans cette crise, c'est que les événements politiques témoignent selon Heidegger d'un profond oubli quant à ce en quoi consistent essentiellement la connaissance et toute pensée comme telle. Reprenant des thèmes véhiculés déjà par une branche de la philosophie allemande du XIXe siècle qui était fermement critique de la culture démocratique en général et du monde universitaire en particulier ${ }^{8}$, Heidegger affirme que la connaissance ne serait maintenant comprise que de façon purement instrumentale, technique : voilà pourquoi selon lui «l'essence de la science» est "vidée et usée9». La pensée et la connaissance, malmenées par la «théologie chrétienne aussi bien qu'après (par) la pensée mathématiquement technique des Temps modernes ${ }^{10}$ ", errent loin de leur origine et de leur grandeur destinale. Heidegger croit fermement qu'une mission salvatrice pour la pensée occidentale est inscrite dans le destin du peuple allemand - et c'est pour cette raison qu'il affirme avoir accepté le rectorat de l'université de Fribourg, afin de prendre part au renouveau spirituel de l'Allemagne et de l'Occident au moyen d'une rénovation interne de l'université allemande.

Heidegger lit essentiellement la crise qui secoue l'Europe comme un signe de la décadence spirituelle de l'Occident. Sur ce point, il serait en accord avec les diagnostics de Nietzsche, de Spengler et de Jünger, par exemple. Mais ce qui fait notamment la spécificité de la lecture heideggerienne, c'est qu'elle reconnaît dans la crise européenne le moment historique de la réalisation d'une mission spirituelle qui est impartie à l'Allemagne de par le lien profond entre l'esprit allemand et l'esprit de la Grèce classique. Cette mission spirituelle définit la tâche politique du peuple allemand. II est important de noter que la position de Heidegger repose ainsi sur un présupposé qui n'est pas discuté dans le Discours de rectorat, à savoir le lien intime entre l'esprit allemand et l'esprit grec. De par sa parenté avec le berceau de la pensée occidentale, l'Allemagne serait seule à même d'opérer un retour à l'essence de la science, afin de contrer l'état de dépravation de la connaissance, de l'enseignement, de la culture et de l'esprit en Europe. Or le Discours de rectorat affirme que l'essence de la science est l'unité de trois savoirs : la connaissance du peuple, la connaissance du destin de l'État et la connaissance de la mission spirituelle du peuple. Heidegger assure que l'unité de ces trois savoirs forme «l'essence originale et pleine de la science dont la mise en 
œuvre est (la) tâche ${ }^{11}$ » des professeurs et des étudiants - soit la tâche propre de l'université allemande. Pour être réalisée, cette tâche demande que l'université allemande, et principalement les étudiants - «la plus jeune force de notre peuple ${ }^{12}$ », affirme Heidegger -, réponde à l'injonction qui est adressée à l'Allemagne à la fois par le moment historique de la crise européenne et par la filiation entre l'esprit allemand et l'esprit grec. Pour accomplir cette mission, les Allemands doivent vouloir l'essence de la connaissance au moyen d'un "retour méditatif sur (eux)-mêmes ${ }^{13}$ » qui les ramènera nécessairement aux sources grecques de la pensée occidentale. En ramenant ainsi la pensée occidentale à sa première jeunesse, le peuple allemand, selon le recteur, s'assurera la grandeur. La tâche politique décrite dans le Discours de rectorat s'adresse à une portion précise du peuple allemand : le monde universitaire, et surtout les étudiants. Quant au peuple allemand en général, "le peuple métaphysique ${ }^{14}$ ", il doit concourir à cette tâche en faisant que la jeunesse allemande se trouve dans les conditions permettant sa réalisation. Notamment, il doit s'assurer que la jeunesse est enracinée dans la nation, car seul cet enracinement peut offrir la triple connaissance nationale qui forme «l'essence originale et pleine de la science ${ }^{15}$ ".

En 1933, Heidegger s'est ainsi aventuré pour la première (et la dernière) fois à présenter ce qui eût été la concrétisation politique de son ontologie fondamentale et de sa philosophie de l'existence. Une éclairante analyse de l'implication politique de la première philosophie de Heidegger a été livrée en 1946 par Karl Löwith. Löwith voulait montrer que la conception de l'existence humaine développée dans Être et temps (1927) mène à une possibilité particulière du politique. Pour ce faire, il exhiba le lien allant du décisionnisme individuel du Dasein au décisionnisme politique du Dasein allemand. Commentant Être et temps, Löwith écrit : «ll suffit de sortir de l'isolement encore à moitié religieux et d'appliquer l'existence "authentique et toujours particulière à chacun" et le Devoir (Müssen) qui en découle à "l'existence particulière allemande" et à son destin historique, pour introduire dans le mouvement général de l'existence allemande l'agitation énergique et vaine des catégories de l'existence ("se décider à soi; se fonder sur soi-même face au néant; vouloir sa propre destinée; s'assumer soi-même") et pour procéder ensuite à la destruction sur le terrain politique ${ }^{16}$ ». Nous ne faisons pas nôtre ici la question de savoir si la pensée heideggerienne correspond intérieurement, conceptuellement, 
au national-socialisme qu'il a cautionné - diverses études ont déjà examiné cette question en profondeur ${ }^{17}$. Nous souhaitons plutôt souligner que la rhétorique du Discours de rectorat témoigne de ce que le philosophe amalgame les catégories philosophiques d'Être et temps et une idéologie de la guerre (Kriegsideologie) répandue dans l'Allemagne des années 193018. Le résultat est un texte d'un genre assez unique dans le corpus heideggerien, qui offre presque un essai d'ontologie politique : la tâche politique et historique du peuple allemand serait déterminée par une décision philosophique dans l'optique de la "problématicité de l'être ${ }^{19}$ ».

Que Martin Heidegger ait affirmé une telle position en un discours politique n'était pas une mince affaire pour le monde philosophique en 1933. Heidegger s'était révélé comme étant le successeur de Husserl et, depuis le débat sur le kantisme en 1929 à Davos, il était devenu le représentant de la «nouvelle philosophie 20 » en Allemagne. Le monde philosophique allemand et Heidegger lui-même ne pouvaient qu'attendre beaucoup de cette nouvelle position. Or, voilà peut-être l'une des raisons faisant que le Discours de rectorat se tient sur plusieurs terrains : programme de rénovation interne de l'université allemande proposé par le nouveau recteur; orientations de pensée livrées par le représentant de la nouvelle philosophie allemande; exhortation des étudiants à l'activisme par un professeur politiquement engagé. Outre la dimension académique du texte, il ne faut donc pas négliger sa dimension activiste : Heidegger cherche en effet à transmettre à un auditoire précis un engouement pour l'engagement politique - et qui plus est, un engagement à travers le service militaire ${ }^{21}$, car ainsi que le rappelle le Discours de rectorat, le travail pour le salut de la nation dans l'Allemagne des années 1930 ne se réalisait pas uniquement dans les rangs de l'université.

Le discours de 1933 témoigne de l'optimisme du recteur et révèle l'espoir qui l'anime, lui qui remarque que la jeunesse "a déjà décidé ${ }^{22}$ ". En effet, le recteur croit que les étudiants allemands se sont mis en quête de renouer avec l'essence de la triple connaissance nationale. Ce qu'il reste donc à accomplir afin de garantir la mission de l'Allemagne, selon Heidegger, c'est d'en assurer les conditions de réalisation, et principalement l'enracinement de la jeunesse dans la nation. Selon le philosophe, c'est le rôle de l'État allemand que d'encadrer la tâche philosophico-politique de la jeunesse. L'État doit reconnaître la mission historique du peuple allemand et garantir les conditions de sa mise en œuvre. Pour ce faire, il est capital que la 
forme de l'État soit spécifiquement allemande et réponde à la particularité de la nation et de l'esprit allemands. II importe pour le philosophe de résoudre l'alternative politique qui déchire l'Occident et d'éviter que l'Allemagne ne se retrouve dans une impasse qui l'oblige à choisir une forme politique étrangère qui lui serait imposée de l'extérieur soit par l'esprit anglo-saxon, soit par l'esprit français, soit par l'esprit russe ${ }^{23}$. Ainsi Heidegger rejoint-il le discours commun à de nombreux intellectuels sous la république de Weimar, qui cherchent alors une "troisième voie" pour l'Allemagne. Cette voie proprement allemande, le recteur croit la reconnaître au début des années 1930 dans le national-socialisme qui présenterait selon lui une possibilité d'aller au-delà de l'alternative "Russie ou Amérique», le communisme et le libéralisme étant des idéologies dominées par la pensée technique et participant de la "décadence spirituelle de la terre ${ }^{24 » .}$ Jamais, peut-être, un philosophe ne fit plus grave erreur de lecture.

Dans une lettre écrite en 1948 à son ancien étudiant Herbert Marcuse, Heidegger témoigne de ses attentes du début des années 1930 : «À propos de 1933 : j'attendais du national-socialisme un renouveau spirituel de la vie tout entière, une réconciliation des antagonismes sociaux et un sauvetage de l'existence occidentale des périls du communisme ${ }^{25}$ ». Ainsi que nous l'avons souligné, c'est, en tant que philosophe, en appliquant les catégories de son ontologie fondamentale et de sa philosophie de l'existence (le Dasein du peuple, la "problématicité de l'être 26 ", etc.) à la compréhension de la crise politique allemande, et, en tant que recteur d'une université allemande, en appelant la jeunesse étudiante à revenir aux sources grecques de la pensée en combattant pour la nation sous le régime nationalsocialiste, que Heidegger espérait participer au "sauvetage de l'existence occidentale".

\section{Le philosophe et le politique}

L'engagement politique de Heidegger suggère un rapport particulier du philosophe au politique. Afin de le dégager, il importe de compléter l'analyse de cet engagement, en soulignant d'abord quelques limites qui lui sont inhérentes, et en examinant ensuite le désengagement du philosophe, soit son retrait du politique suite à l'échec de 1933-1934. Nous pourrons alors remarquer que malgré ce désengagement, des allusions politiques apparaissent en filigrane des textes heideggeriens d'après-guerre. En précisant la teneur de ces allusions, nous pourrons compléter notre analyse de l'engagement 
politique de Heidegger et dégager le rapport du philosophe au politique qu'il suggère.

L'appréciation qu'a donnée Heidegger de son essai d'engagement politique et son rejet futur de tout engagement politique montrent les limites inhérentes à son engagement. Mais d'emblée, il importe de rappeler que l'épisode proprement politique de Heidegger a été bref compte tenu du fait que la carrière du philosophe s'échelonne sur une soixantaine d'années. Après 1934, Heidegger a abandonné toute tentative de donner une forme politique à sa pensée philosophique. II a toujours été laconique par rapport à l'échec de son engagement : à ce sujet l'essentiel de sa position d'après-guerre tient dans les propos qu'il livra en 1966 à la revue d'actualité allemande Der Spiegel, affirmant que l'espoir qui l'animait au début des années 1930 aurait été déçu par les nationaux-socialistes dont la pensée "était beaucoup trop indigente pour parvenir à une relation vraiment explicite avec ce qui arrive aujourd'hui et qui était en route depuis trois siècles 27 ", à savoir la domination du mode de pensée technique comme rapport inclusif au monde. Cette affirmation est loin de former une excuse, et elle rappelle en fait que Heidegger n'a jamais adopté une perspective critique sur son engagement politique. Après 1934, il s'est cantonné au travail philosophique, abandonnant toute velléité de donner une forme politique à sa pensée philosophique. Après une brève mais désastreuse tentative de mener l'interrogation philosophique sur le terrain de la décision politique, Heidegger a cherché à faire une philosophie apolitique. Sa pensée philosophique se serait développée en marge du politique, et ce, suite à l'échec même d'une tentative d'engagement politique.

Voilà qui présente un constat qui demande examen - car le fait qu'il ait débouché sur un rejet de l'expression politique de la philosophie est une dimension essentielle de l'engagement heideggerien. Le rapport du philosophe au politique chez Heidegger est rapidement passé de l'engagement au désengagement. Ses cours des années 1920 et 1930 montraient déjà que le professeur ne s'intéressait pas à la question politique, à laquelle il ne s'arrêtait pour ainsi dire jamais 28 . En 1933, le professeur a entrepris de franchir les limites purement académiques de l'activité philosophique auxquelles il s'était cantonné jusque-là, mais c'était pour peu après considérer cet engagement comme une véritable transgression par rapport à l'activité philosophique. Dès 1945, Heidegger affirme que le philosophe ne doit 
pas se mêler de politique29. Dans ses textes d'après-guerre, il rejette toute question pratique ${ }^{30}$ comme relevant de l'inessentiel, voire de l'impossible, et c'est une position qu'il conserve jusque dans ses derniers écrits, ainsi que le montrent ces propos de 1966 : «Pour autant que je puisse voir, un individu n'est pas mis en mesure par la pensée d'avoir sur le monde dans sa totalité une vue si pénétrante qu'il puisse donner des indications pratiques sur ce qu'il faut faire (...). C'est trop demander à la pensée (...). (L)a pensée doit s'interdire de donner la réponse du jour aux questions pratiques portant sur sa conception du monde ${ }^{31}$ ». Pour Heidegger, le problème philosophique fondamental n'est pas formulé par le vivre-ensemble, mais plutôt par l'événement majeur de l'histoire de la pensée occidentale qu'est l'achèvement de la métaphysique de la subjectivité. Cet événement rendrait possible un retour à une forme de pensée proche de celle des présocratiques et de celle qui se fait entendre à nouveau chez certains poètes, tels René Char ou Rilke. II s'agit donc pour le philosophe d'œuvrer à la rénovation de la civilisation par la voix de la poésie plutôt que par la voie politique : Heidegger estime que la pensée ne doit pas s'aventurer dans des domaines qui sont inessentiels par rapport à la tâche primordiale de penser l'être dans son éternel jeu de voilement et de dévoilement.

C'est en montrant que le politique n'est pas l'affaire propre de la pensée, que Heidegger justifie son refus d'avancer des affirmations politiques positives. Aussi ses rares propos politiques prennent-ils la forme de l'aveu d'ignorance, par exemple lorsqu'il dit ne pas être en mesure de savoir quel régime ou quelle forme d'organisation sociale et de gestion économique des ressources seraient adaptés à l'époque contemporaine : "C'est pour moi aujourd'hui une question décisive de savoir comment on peut faire correspondre en général un système politique à l'âge technique et quel système ce pourrait être. Je ne sais pas de réponse à cette question 32 ». Selon Heidegger, seule une révélation pourra montrer la voie politique viable - et il n'est pas même certain qu'une telle révélation adviendra ${ }^{33}$. Aussi le philosophe doit-il éviter les questions politiques qui ne peuvent que le détourner du problème philosophique fondamental et l'entrainer dans l'erreur : selon Heidegger, celui qui s'intéresse aux questions pratiques risque de voir sa réflexion fondamentale minée par les formes de la pensée politique.

C'est un tel raisonnement qui sous-tend l'appréciation que le philosophe a donnée de son engagement politique des années 1930. 
Selon ses propres mots, en s'éloignant de la méditation des questions essentielles de la philosophie pour réfléchir à leurs dérivés pratiques, le philosophe aurait «renoncé au métier proprement dit de penser, en faveur d'une action en tant qu'administrateur ${ }^{34}$ », s'égarant ainsi dans des considérations secondaires. Heidegger pensait-il à l'épisode de son rectorat lorsqu'il affirma trente ans plus tard : «ll se peut bien (...) que le chemin d'une pensée conduise aujourd'hui à se taire, pour empêcher que la pensée ne soit bazardée en l'espace d'un an35»? La pensée de l'être, la véritable philosophie selon Heidegger, est plus importante que «d'impuissantes déterminations de fins qui se limitent à la seule sauvegarde de l'humain ${ }^{36}$ ". Tout ce qui touche l'organisation de la société humaine est secondaire pour le philosophe par rapport à la tâche primordiale de méditer l'être.

La position tenue par Heidegger dans l'après-guerre suggère que son rejet du politique en philosophie serait la conséquence directe de l'échec de son engagement politique de la première moitié des années 1930. Malgré ce rejet, toutefois, des allusions politiques ponctuent les textes que Heidegger a écrits après l'épisode de son engagement. Bien qu'elles soient rares et toujours succinctes, ces allusions précisent le rapport du philosophe Heidegger à la cité : elles montrent que malgré son rejet du politique en philosophie et son refus de donner une forme politique à sa pensée après 1934, Heidegger affirme une position politique. Celle-ci se dessine dans quelques affirmations que l'on peut tirer des textes des années 1930-1950. Peu après sa démission du rectorat, dans son cours de l'été 1935 , Heidegger affirme que la "Russie et l'Amérique sont toutes deux, au point de vue métaphysique, la même chose; la même frénésie sinistre de la technique déchaînée, et de l'organisation sans racines de l'homme normalisé37». II précise : «'Europe se trouve dans un étau entre la Russie et l'Amérique, qui reviennent métaphysiquement au même quant à leur appartenance au monde et à leur rapport à l'esprit38». Quelques années plus tard, dans une conférence de 1938, il affirme : «Ce n'est que là où l'homme est déjà, par essence, sujet, qu'est donnée la possibilité de l'aberration dans l'inessentiel du subjectivisme au sens de l'individualisme. Mais ce n'est également que là où l'homme reste sujet que la lutte expresse contre l'individualisme et pour la communauté en tant que champ et but de tout effort et de toute espèce d'utilité a seulement un sens ${ }^{39}$ '. Enfin, dans une note de 1951, il écrit : «partout (...), avant toutes les différences nationales, (l')uniformité de l'étant entraîne l'uniformité de la direction, pour 
laquelle toutes les formes politiques ne sont plus qu'un instrument de direction parmi les autres ${ }^{40}$ '.

Ces affirmations sont révélatrices quant à la position politique de Heidegger. L'on peut d'emblée noter que peu importe l'État, le régime ou l'idéologie dominante, Heidegger considère que l'Occident est décadence, uniformité grandissante et vide spirituel, "organisation sans racines de l'homme normalisé41". C'est à cet égard qu'il qualifie encore en 1966 toute idéologie politique de «demi-mesure» incapable d'«entrer suffisamment en relation avec l'être de la technique" et de mettre "en question (le) monde technique ${ }^{42}$ ». Le philosophe tient toute idéologie politique occidentale comme une excroissance de la domination de la conception de l'être humain comme subjectivité et, en ce sens, comme maître cherchant à asseoir sa puissance sur la totalité de ce qui est sur terre. Or la critique heideggerienne de la métaphysique de la subjectivité entraîne un dualisme entre volonté et destin et une critique concomitante de ce qui est institué sur l'ordre de la volonté43. Pour Heidegger, l'ontologique détermine le philosophique : la pensée doit méditer l'être. Aussi le philosophe refuse-t-il à la pensée toute prétention d'établir ou de justifier l'institution d'un régime politique sur l'ordre de la volonté. Comme un ordre politique est nécessairement engagé dans une lutte pour la domination planétaire des ressources, un régime, quel qu'il soit, ne sera du coup qu'une forme parmi d'autres d'une seule et même volonté de maîtrise de l'étant, ou de l'ensemble de ce qui est. De là découle, selon Heidegger, l'uniformité métaphysique des systèmes politiques - et de là découle, encore une fois, le désintérêt du philosophe pour les questions politiques. Refusant toute justification d'un pouvoir ou d'un contrôle humains sur l'étant, la pensée heideggerienne, loin de l'action, se veut plutôt contemplation, parole poétique et non parole politique. Or que serait une politique instituée sur l'ordre du destin plutôt que sur l'ordre de la volonté? Que serait une "politique de l'être"? Les limites du présent travail ne nous permettent pas d'engager ici une interprétation politique de la pensée de l'être. Qu'il nous soit simplement permis de noter que Heidegger n'a jamais cherché à répondre à ces questions, limitant, ainsi que nous l'avons souligné, ses affirmations à l'aveu d'ignorance ou à l'attente d'une révélation. Aussi la pensée politique heideggerienne est-elle purement négative.

Ce constat complète le rapport du philosophe au politique chez Heidegger. L'analyse que nous avons menée jusqu'à présent 
distingue trois dimensions à ce rapport, la première étant celle de l'engagement politique du philosophe : le philosophe devenu recteur voit dans la crise européenne le moment historique où l'affirmation de la nation allemande pourra, par un retour aux sources grecques de la pensée, régénérer la civilisation occidentale, et définit l'université allemande comme le lieu de cette affirmation philosophico-politique. La seconde dimension est celle du désengagement ou du retrait du politique par le philosophe, par suite de la déception qu'ont entraînée chez Heidegger l'indigence et la pauvreté de vision des nationauxsocialistes face à la crise européenne. Cette déception a mis un terme à l'espoir qui animait le philosophe en 1933 et dont témoigne le Discours de rectorat. Enfin, la troisième dimension est celle de la pensée politique négative qui se déploie au fil de l'œuvre philosophique du "second" Heidegger, de la deuxième moitié des années 1930 à la fin des années 1960. Malgré son désengagement, Heidegger conserve une position politique négative qui s'exprime par la voix d'une critique des formes politiques de la métaphysique de la subjectivité.

Ce triple rapport du philosophe Heidegger au politique montre d'une part un revirement entre l'engagement du philosophe et son désengagement, et d'autre part un paradoxe entre cette volonté de retrait total et la constance d'une position politique, fermement critique de la modernité occidentale. II va sans dire, le rapport du philosophe au politique chez Heidegger est complexe. Toutefois, nous ne souhaitons pas nous limiter à la seule tâche de dégager ce rapport dans sa complexité. Afin de terminer notre analyse, nous voulons examiner les sources philosophiques du rapport de Heidegger au politique.

\section{Une double filiation philosophique}

Nous croyons que la filiation philosophique du rapport du philosophe au politique chez Heidegger est double, remontant d'une part à Jünger et d'autre part à Nietzsche. Nous examinerons cette double filiation en soulignant d'abord les apports de la pensée de l'essayiste, puis les apports de la philosophie nietzschéenne au rapport heideggerien du philosophe à la cité.

Dans les années 1930, Heidegger s'est intéressé aux premiers essais de son contemporain Ernst Jünger, notamment La mobilisation totale (1930) et Le Travailleur (1932). Dans son texte de 1945 sur le rectorat, il souligne l'importance qu'a eue sa lecture de Jünger pour le 
développement de sa pensée 44 . Deux aspects inextricablement liés de la pensée jüngérienne sont repérables chez Heidegger dans la conception qu'il se fait de la crise européenne : le diagnostic et le vocabulaire qui l'exprime. Dans La mobilisation totale, Jünger avance que la Première Guerre mondiale a révélé que le nouveau caractère de l'étant, ou de la totalité de ce qui est, est la mobilisation, dont le principe décisif est la «disponibilité à être mobilisé45» : ancien soldat du front, l'essayiste conçoit l'étant dans son ensemble comme disponibilité sans fin à être mobilisé pour le simple but de libérer toujours plus d'énergie. La mobilisation entraînerait partout et sans cesse des déploiements de force "dont le signe distinctif est d'être dépourvus de finalité 46 ». Selon Jünger, la mobilisation totale est dorénavant le caractère fondamental de l'être, elle est la forme que l'époque imprime à l'existence.

Heidegger décrit l'époque contemporaine en des termes qui font référence aux descriptions de Jünger et aux métaphores qu'il emploie. Le philosophe fait notamment état du "parachèvement et de l'exploitation organisée requis par l'époque" et de "la sphère vive des

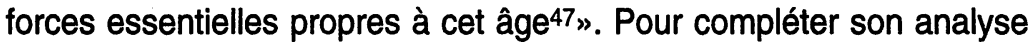
du nouveau paradigme de la mobilisation totale, Jünger affirmait que "si la logique humaine veut garder quelque efficacité, il lui faudra suivre un cours parallèle" à la mobilisation totale 48 . Heidegger fait écho à l'essayiste lorsqu'il écrit que comme la réalité "consiste dans l'uniformité du calcul traduisible en plans, il faut que l'homme lui aussi entre dans l'uniformité, s'il veut rester en contact avec le réel49". Toutefois, alors que Jünger appelait ainsi une participation voulue au processus de mobilisation totale, Heidegger décrit plutôt un état de fait qu'il s'agit de critiquer ${ }^{50}$. Mais par-delà les divergences quant aux prescriptions d'action entraînées par le paradigme de la mobilisation, il faut souligner que le diagnostic du philosophe recoupe celui de l'essayiste. C'est en dialogue avec Jünger que Heidegger mène sa réflexion sur la nouvelle époque qui se dessine dans les années 1930, et c'est souvent dans des termes jüngériens qu'il décrit la crise en cours, comme lorsqu'il affirme que «le travail (...) accède aujourd'hui au rang métaphysique de cette objectivation inconditionnelle de toutes les choses présentes, qui déploie son être dans la volonté de volonté51 ». Dans les premiers essais d'Ernst Jünger, Heidegger a par ailleurs pu lire l'idée qu'au principe de tout régime politique se déploie une volonté de domination des ressources, une même dynamique de la puissance pour la puissance qui tend à s'étendre à l'ensemble de la planète, signant ainsi l'uniformisation des modes de vie. Dans cette 
optique, pour Heidegger comme pour Jünger, peu importe qu'un État se définisse comme régime démocratique ou comme régime fasciste, une seule idéologie, celle de la pure puissance, détermine tout régime politique institué sur l'ordre de la volonté52.

Si l'apport de la pensée jüngérienne chez Heidegger est manifeste, il n'est toutefois pas suffisant pour rendre compte de la forme qu'a prise l'engagement politique heideggerien, ainsi que du retrait du philosophe par rapport au politique. C'est pourquoi il nous faut nous tourner vers Nietzsche, auquel Heidegger s'est particulièrement intéressé dans les années 1930, lui consacrant de nombreux cours et conférences dès 1936 et jusqu'aux années 1950. Nous soulignerons quatre apports de la pensée nietzschéenne à la détermination du rapport du philosophe au politique chez Heidegger.

Le premier apport est l'idée d'une rénovation interne de l'université allemande. Si cette thématique est au cœur du Discours de rectorat, c'est aussi l'un des projets capitaux du jeune Nietzsche que d'effectuer un travail profond sur l'université, ainsi qu'en témoignent notamment ses cinq conférences prononcées à l'université de Bâle à l'hiver 1872, "Sur l'avenir de nos établissements d'enseignement". La position de Nietzsche est liée à une critique de l'enseignement universitaire et de l'état académique de la connaissance en Allemagne, qui est largement inspirée des charges portées par Schopenhauer dans son pamphlet Contre la philosophie universitaire (1851). Ainsi Heidegger se place-t-il d'emblée dans un courant de la philosophie allemande qui souhaite amener l'université à renouer avec l'idéal d'une Bildung pour faire contrepoids à la surspécialisation qui caractérise l'éducation universitaire et au morcellement de la connaissance qui en résulte. "Le pouvoir formateur de notre enseignement supérieur n'a jamais été ni si bas ni si faible ${ }^{53}$ ", écrivait Nietzsche dans son tout premier livre en 1872. Voilà qui lui faisait écrire à son ami Erwin Rohde : "tout mon travail intellectuel est consacré à l'avenir de nos établissements d'enseignement ${ }^{54}$ ». C'est dans cette même optique d'une critique culturelle qui passe par un programme d'amélioration de l'université allemande, que Heidegger situe sa tâche en tant que recteur d'une université allemande dans un moment de crise politique et culturelle. L'engagement politique de 1933 est intimement lié au projet d'une réforme de l'université allemande par la philosophie qui, pour Schopenhauer et Nietzsche comme pour Heidegger, a un rôle essentiel à jouer dans la formation de l'esprit. 
Le deuxième apport de la pensée nietzschéenne à la détermination du rapport du philosophe au politique chez Heidegger, est la nécessité pour l'esprit allemand d'effectuer un retour aux Grecs. Heidegger annonce dans son Discours de rectorat que ce retour est un passage obligé de la revivification de la connaissance, ainsi qu'une étape-clef du mouvement par lequel l'esprit allemand atteindra la grandeur destinale qui lui est promise. II n'est pas étonnant de voir l'idée d'un retour aux Grecs à l'œuvre dans les écrits d'un professeur de philologie classique au XIX ${ }^{\theta}$ siècle, tel que l'était le jeune Nietzsche, titulaire d'une chaire à l'université de Bâle de 1869 à 1879. Nietzsche avait notamment développé cette idée en 1872 dans La naissance de la tragédie, en lançant une exhortation afin que l'esprit allemand "vive d'heureuses retrouvailles avec lui-même", la seule condition à cette auto-affirmation étant que le peuple allemand "se mette, sans la moindre hésitation, à l'école de ce peuple, dont c'est déjà la plus haute des gloires et un rare privilège que d'être capable d'en recevoir les leçons : je veux parler des Grecs 55 ". Ce qui se présente toutefois dans le Discours de rectorat comme le postulat d'une parenté entre l'esprit grec et l'esprit allemand, trouve chez Nietzsche une justification de type esthétique. Le philologue fonde la nécessité d'un retour aux Grecs sur ce qu'il estime être la supériorité du sentiment esthétique grec (qui implique pour lui la supériorité de la compréhension grecque de l'existence humaine) et de l'œuvre d'art suprême qu'est la tragédie grecque, œuvre et sentiment qu'il s'agirait pour l'Allemagne de prendre en exemple (comme le firent par exemple Goethe et Schiller, que Nietzsche admire) afin d'encourager un effort culturel qui serait salvateur pour l'esprit allemand. Bien que Heidegger ne suive pas en 1933 cette voie précise d'une esthétisation du projet politique allemand 56 , la forme que prend l'engagement politique de sa philosophie est nettement marquée par l'importance qu'accorde Nietzsche à un retour aux Grecs pour la régénération de la civilisation allemande. À lire la justification philosophique du projet politique qui se dessine dans le Discours de rectorat, l'on pourrait croire que le "nouveau philosophe» et recteur faisait sienne cette injonction que Wagner adressait à Nietzsche en 1872 par l'entremise d'une lettre ouverte : "Ce que nous attendons de vous ne peut être que la tâche de toute une vie, et de la vie d'un homme tel que nous en avons le plus urgent besoin, et tel que vous vous annoncez à tous ceux qui demandent aux sources les plus pures de l'esprit allemand - l'intime et profond sérieux qu'il met dans tout ce qu'il entreprend - des 
éclaircissements et des indications sur ce que doit être la culture allemande pour pouvoir aider la nation régénérée à atteindre ses buts les plus nobles ${ }^{57}$ ".

La pensée nietzschéenne présente par ailleurs un apport à la détermination du rapport du philosophe au politique chez Heidegger quant à la thématique d'une "grande politique» pour l'Europe. Heidegger n'emploie pas ces termes de Nietzsche que l'on trouve dans Par-delà bien et mal, mais l'idée d'une grande politique menée par l'Allemagne à l'échelle européenne pour le salut de l'Occident est au cœur du Discours de rectorat. Heidegger aurait assigné à la philosophie la tâche d'une grande politique alors que Nietzsche, lui, maintenait une distance, voire même un fossé, entre le philosophe et le politique. Nietzsche estimait que la grande politique nécessitait une nouvelle sorte de "chefs", mais il n'affirmait pas que ces chefs devaient être des philosophes, ni que les philosophes aient à se charger de la domination politique ou même plus simplement de la détermination des politiques pratiques. Heidegger semble avoir réuni en un seul programme ce que Nietzsche présentait comme étant les tâches de deux types d'hommes : d'une part le travail propre au philosophe, à savoir une "grandiose entreprise d'éducation et de sélection" pour "mettre fin à l'effroyable règne du non-sens et du hasard qui s'est appelé "histoire" jusqu'à présent58", et d'autre part le travail proprement politique de donner une direction à l'Europe ${ }^{59}$. Ces deux tâches ne peuvent être celles d'un seul homme, car selon Nietzsche, le philosophe doit se tenir écarté du politique. Or voilà une distinction nietzschéenne que Heidegger n'aurait suivie qu'après l'échec de son engagement politique.

C'est ce qui nous amène au quatrième apport de la pensée nietzschéenne à la détermination du rapport du philosophe au politique chez Heidegger, à savoir le besoin pour le philosophe de se tenir à l'écart du politique. Dans son ouvrage de 1886, Nietzsche présente la politique pratique comme une «souillure60». À l'opposé de cette "domination grossière", une forme de domination plus «raffinée» serait réservée au philosophe. Il aurait ainsi des tâches plus hautes que la politique pratique, «et plus que royales61». La domination raffinée propre au philosophe nécessiterait qu'il mène une vie retirée et contemplative, entourée de disciples 62 : ainsi pourrait-il s'assurer des conditions lui permettant de mener son œuvre éducative, sélective et créatrice de "la destination et la finalité de I'homme ${ }^{63}$ ». Heidegger a retenu la mise en garde de Nietzsche quant 
au caractère second des questions politiques pour le philosophe, mais seulement après son essai d'engagement politique. En tentant de mener l'interrogation philosophique sur le terrain de la décision $p$ olitique, aurait-il fait l'expérience du danger qui est selon Nietzsche l'élément propre du philosophe?

Considérant «les choses de haut en bas » dans la tradition d'une pensée ésotérique 64 , croyant fermement, dans la lignée de Schopenhauer, à un ordre hiérarchique de la nature et des êtres humains, le philosophe, selon Nietzsche, doit être masqué lorsqu'il se retire du jardin où il discute parmi ses semblables, car il mène une vie imprudente et se met continuellement en danger65. Or si Nietzsche a passé la moitié de sa vie d'écrivain à vivre en philosophe errant, retiré du monde académique comme du monde politique, Heidegger, en tant que professeur d'université et ancien recteur nazi, a vécu les conséquences d'une prise de position politique extrémiste. L'erreur politique de Heidegger fut immense, lui qui affirmait toujours en 1945 avoir "vu dans le mouvement parvenu au pouvoir (en 1933) une possibilité de rassembler et de renouveler le peuple (allemand) depuis l'intérieur; un chemin pour trouver sa détermination historique et occidentale66». Pour sa démission du rectorat, il fut surveillé par le pouvoir nazi; pour son engagement national-socialiste, il fut sommé de se justifier devant la commission d'épuration et interdit d'enseignement jusqu'en 1956. Heidegger a vécu les tensions qui peuvent naître entre la cité et une certaine idée de la philosophie. Cette idée de la philosophie, qui ne prend qu'à risque la forme d'une éthique ou d'une esthétique ${ }^{67}$, était celle déjà de Schopenhauer et de Nietzsche - et elle serait celle, aussi, d'Ernst Jünger, lui qui affirmait en 1981 : "Nietzsche fut l'un des premiers à avoir pressenti qu'un nécessaire renversement des valeurs allait un jour se produire, mais, quant à moi, je me garderai bien de me risquer à formuler une éthique qui correspondrait à la figure du Travailleur. Mieux vaut se taire à ce sujet ${ }^{68}$...".

C'est sur cette voie d'un mutisme qui en vérité en dit long, que s'est dirigé Heidegger suite à l'échec de son engagement politique. Du constat (en partie jüngérien) de la dépravation de la civilisation occidentale, au projet (en partie nietzschéen) de son sauvetage par la régénération de l'esprit allemand au moyen d'une rénovation interne de l'université allemande et d'un retour spirituel aux Grecs, le rapport du philosophe au politique chez Heidegger a changé du tout au tout, pour se faire retrait, rejet, aveu d'ignorance et, le plus souvent 
possible, silence69.

L'étude que nous avons menée en ces pages a voulu montrer que l'engagement politique de Heidegger offre à la philosophie l'occasion d'une importante réflexion sur le rapport parfois ambigu, et souvent problématique, qu'elle entretient avec le politique. L'exemple de Heidegger peut servir de cas-limite de l'engagement politique en philosophie. En ayant repris des thématiques propres à une certaine idée de la philosophie, foncièrement aristocratique - une idée courante à l'époque classique mais aussi dans la modernité récente, comme le montre le XIXe siècle de Schopenhauer et de Nietzsche ou, plus près de nous, l'entre-deux-guerres de Jünger et de Heidegger -, en ayant repris, donc, une idée aristocratique de la philosophie, et en ayant tenté de lui donner une forme politique, Heidegger a suggéré que cette idée de la philosophie ne pouvait s'aventurer sur le terrain politique ou éthique qu'en risquant la philosophie elle-même. Heidegger en a conclu que la philosophie ne devait en aucun cas se mêler de politique, sous peine de nuire à la vie même du philosophe et, du coup, à l'exercice de l'activité philosophique essentielle et primordiale, selon Heidegger, qu'est la méditation de l'être. Or c'est là la conclusion qu'il tire d'une certaine idée de la philosophie, qui n'épuise pas toutes les formes possibles de l'activité philosophique.

Mais que penser aujourd'hui d'une idée de la philosophie si étrangère aux acquis de la modernité politique, dès lors que l'on sait combien ces acquis sont fragiles et susceptibles de s'écrouler? Cette idée de la philosophie, et notamment l'exemple qu'en offre Heidegger, peut amener la pensée occidentale à poursuivre l'importante réflexion sur le rapport qu'elle entretient à la vie politique et sociale. L'exemple de Heidegger rappelle qu'il n'est pas vrai que toutes les positions philosophiques peuvent trouver une forme politique ou éthique viable. II y a des écueils sur lesquels la philosophie est vouée à s'échouer continuellement à moins qu'elle ne maintienne un travail d'autoréflexion qui ne peut qu'être profitable à une tâche plus générale de la philosophie, et qui est celle de former la pensée critique et de former des citoyens.

L'exhortation lancée par Heidegger dans son Discours de rectorat à la jeunesse allemande ne visait aucunement à former des citoyens éclairés dotés d'une pensée critique, capables d'appréciations plurielles des événements qui marquent la vie d'un individu ou celle d'une collectivité. L'erreur de Heidegger fut immense. Mais Heidegger était philosophe, et ainsi que nous l'avons montré, sa posture 
philosophique et politique témoigne des apports d'une certaine tradition philosophique. Pour cette raison, il importe que les livres de Heidegger demeurent sur les rayons de philosophie, afin que la philosophie soit à même de repérer lesquelles de ses formes ne sont pas viables politiquement et qu'elle connaisse ses erreurs, ses risques, ses écueils. Nietzsche affirmait que le danger est l'élément du philosophe. Or Heidegger complète cette idée en montrant que le philosophe peut lui-même être un danger pour la philosophie.

Heidegger présente à la philosophie une question à laquelle elle est périodiquement confrontée, à savoir s'il est possible pour le philosophe de se détourner du politique pour se concentrer sur la tâche de faire œuvre en philosophie. Malgré les efforts qu'il aura déployés en ce sens, Heidegger semble laisser la philosophie sur le constat qu'il est impossible de mener une philosophie apolitique. La responsabilité politique du philosophe n'en est que plus immense.

\section{Martine Béland \\ École des hautes études en sciences sociales, Paris Et Centre canadien d'études allemandes et européennes, Montréal}

1. Une version préliminaire de ce texte fut présentée dans le cadre du colloque "Activité critique et idéologies. Trajectoires d'intellectuels en Europe au $x x^{e}$ siècle» qui eut lieu à l'Université de Montréal en septembre 2005. L'auteure souhaite remercier Kevin Hébert de lui avoir donné l'occasion de préparer ce texte, ainsi que Jean-Philippe Warren et Augustin Simard pour leurs commentaires fort utiles sur une version antérieure de cet article. Elle tient de plus à remercier le Conseil de recherches en sciences humaines du Canada pour son appui lors de ses recherches.

2. C'est ce que remarqua Karl Löwith dès 1946, en signant un article dans Les temps modernes sur la dimension politique de la philosophie heideggerienne ("Les implications politiques de la philosophie de l'existence chez Heidegger"; trad. J. Rovan, vol. 2, $\left.n^{\circ} 14\right)$.

3. Cf. L. Ferry et A. Renaut, Heidegger et les modernes (Paris, Grasset, 1988); et A. Münster, Heidegger, la "science allemande" et le national-socialisme. Suite d'une polémique... (Paris, Kimé, 2002).

4. Cf. E. Faye, Heidegger. L'introduction du nazisme dans la philosophie (Paris, Albin Michel, 2005).

5. Cf. N. Tertulian, «Histoire de l'être et révolution politique. Réflexions sur un ouvrage posthume de Heidegger" (Les temps modernes, vol. $45, n^{\circ} 523,1990$ ) et "La pensée de Heidegger sous le regard démystificateur d'un sociologue» (in J. Bidet et J. Texier [dir.], Actuel Marx, $\mathrm{n}^{\circ} 20$ : Autour de Pierre Bourdieu, Paris, P.U.F., 1996, p. 39-45); J.-M. Palmier, Les écrits politiques de Heidegger (Paris, L'Herne, 1968). 
6. Cf. D. Losurdo, Heidegger et l'idéologie de la guerre (trad. J.-M. Buée, Paris, P.U.F., 1998) et P. Bourdieu, L'ontologie politique de Martin Heidegger (Paris, Minuit, 1988 [1975]).

7. Ce texte fut d'abord traduit en français en 1982 sous le titre L'auto-affirmation de l'université allemande (trad. G. Granel, éd. T.E.R.), puis en 1983 par François Fédier sous le titre "L'université allemande envers et contre tout elle-même" (Le Débat, vol. 27, p. 90-97). C'est à cette seconde traduction que nous nous référons.

8. Voir notamment le pamphlet de Schopenhauer Contre la philosophie universitaire (1851) et les conférences de Nietzsche Sur l'avenir de nos établissements d'enseignement (1872).

9. "L'université allemande», p. 92.

10. Ibid., p. 92.

11. Ibid., p. 95.

12. Ibid., p. 97.

13. Ibid., p. 91. Notons que ce décisionnisme est absent des textes post-rectorat (ou des textes suivant le "Tournant») de Heidegger.

14. Introduction à la métaphysique, cours de l'été 1935 (trad. G. Kahn, Paris, Gallimard, coll. Tel, 1980), I, p. 35.

15. "L'université allemande», p. 95. Heidegger maintiendra jusque dans ses derniers textes l'importance de l'enracinement, ainsi par exemple en 1966 : "toute chose essentielle et grande a pu seulement naître du fait que l'homme avait une patrie (Heimat) et qu'il était enraciné dans une tradition" (Réponses et questions sur l'histoire et la politique, entretien livré à l'hebdomadaire allemand Der Spiegel en 1966, trad. J. Launay, Paris, Mercure de France, 1977, p. 47).

16. Löwith, op. cit., p. 348.

17. Voir entre autres le livre de L. Ferry et d'A. Renaut (op. cit.). Pour une analyse moins nuancée mais représentative d'un type de commentaire de Heidegger qui refait continuellement surface, voir le livre d'A. Münster (op. cit.).

18. Dans Heidegger et l'idéologie de la guerre, D. Losurdo compte au nombre des topoi de la Kriegsideologie présents chez le jeune Heidegger "la communauté" (la Gemeinschaft, pensée en opposition à la Gesellschaff), "la fidélité" et "le destin" (cf. Losurdo, op. cit., ch. 2). A cet égard, voir aussi Habermas qui souligne que la pensée heideggerienne des années 1920 et 1930 témoigne d'une idéologie qui "se composait de la vision élitiste que les universitaires avaient d'eux-mêmes, d'un fétichisme de l'esprit, d'une idolâtrie de la langue maternelle, du mépris de toute réalité sociale, de l'absence complète de toute perspective sociologique (...), d'une polarisation entre sciences naturelles et sciences de l'esprit” (J. Habermas, Martin Heidegger. L'œuvre et l'engagement, trad. R. Rochlitz, Paris, Cerf, 1988, p. 22).

19. "L'université allemande», p. 95.

20. C'est l'appréciation qu'en suggère Marcuse lorsqu'en 1974 il se remémore l'esprit des années 1928-1932 qu'il passa à l'université de Fribourg auprès de Heidegger : "We saw in Heidegger what we had first seen in Husserl, a new beginning, the first radical attempt to put philosophy on really concrete foundations - philosophy concerned with the human existence, the human condition, and not merely with abstract ideas and principles" ("Heidegger's Politics : An Interview with Herbert Marcuse by Frederick Olafson", Graduate Faculty Philosophy Journal, vol. 6, no 1, 1977, p. 28). 


\section{Martine Béland}

21. Heidegger écrit que c'est "la problématicité de l'être en général qui extorque au peuple travail et combat" ("L'université allemande», p. 95). Voilà un exemple probant de la manière toute équivoque dont Heidegger amalgame la philosophie d' tre et temps et la Kriegsideologie dans son Discours de rectorat.

22. "L'université allemande», p. 97.

23. Notons que cette thématique de la voie politique allemande est plus explicite dans le cours de l'été 1935, Introduction à la métaphysique, que dans le Discours de rectorat de 1933.

24. Heidegger, Introduction à la métaphysique, I, p. 49; cf. aussi iv, p. 202.

25. Lettre datée du 20 janvier 1948, citée par N. Tertulian, "Histoire de l'être et révolution politique", op. cit., p. 119.

26. "L'université allemande», p. 95.

27. Réponses et questions, p. 61-62.

28. Sa dissertation d'habilitation (1916) porte sur Duns Scot, et ses premiers cours examinent Platon (1924-1925 : Le Sophiste), Aristote (1931 : Métaphysique), Kant (1927, 1929 : Critique de la raison pure), Hegel (1930 : Phénoménologie de l'esprit), ainsi que les concepts fondamentaux de la métaphysique $(1923,1927$, 1929-1932). En contrepartie, aucune trace d'intérêt chez lui pour le socialisme (qui intéressait pourtant Nietzsche), la doctrine de l'État et les philosophes politiques (Locke, Hobbes, Rousseau, Machiavel, etc.). Même les écrits de Marx ne l'ont pas intéressé : à notre connaissance, on ne trouve dans les textes de Heidegger qu'une seule allusion à Marx, en 1946, judicieusement insérée (compte tenu de l'époque) dans la Lettre sur l'humanisme (trad. R. Munier, éd. bilingue, Paris, Aubier, 1964, p. 103).

29. Cf. le texte de 1945 "Le rectorat 1933-1934. Faits et réflexions", que nous citons plus loin.

30. II faut dire «pratique» et non pas seulement «politique», du fait du rejet intégral de la pensée pratique en faveur d'une pensée méditative dans la Lettre sur l'humanisme.

31. Réponses et questions, p. 70-72.

32. Ibid., p. 42.

33. Cf. Réponses et questions, p. 48-49 : «la philosophie ne pourra pas produire d'effet immédiat qui change l'état présent du monde. Cela ne vaut pas seulement pour la philosophie, mais pour tout ce qui n'est que préoccupations et aspirations du côté de l'homme. Seulement un dieu peut encore nous sauver. II nous reste pour seule possibilité de préparer dans la pensée et la poésie une disponibilité pour l'apparition du dieu ou pour l'absence du dieu dans notre déclin".

34. Heidegger, "Le rectorat 1933-1934. Faits et réflexions", 1945 (trad. F. Fédier, Le Débat, vol. 27, 1983), p. 86.

35. Réponses et questions, p. 57. Cf. aussi la Lettre sur l'humanisme : «Peut-être alors le langage exige-t-il beaucoup moins l'expression précipitée qu'un juste silence" (p. 113).

36. Heidegger, Langue de tradition et langue technique, conférence de 1962 (trad. M. Haar, Paris, Lebeer-Hossman, 1990), p. 31.

37. Introduction à la métaphysique, I, p. 49.

38. Ibid., I, p. 56. 
39. Heidegger, "L'époque des "conceptions du monde" (in Heidegger, Chemins qui ne mènent nulle part, trad. W. Brokmeier, Paris, Gallimard, coll. Tel, 1986), p. 121.

40. Heidegger, "Dépassement de la métaphysique», notes de 1936 à 1946 (in Heidegger, Essais et conférences, trad. A. Préau, Paris, Gallimard, coll. Tel, 1996), § 26 (seul § écrit en 1951), p. 112.

41. Introduction à la métaphysique, I, p. 49.

42. Réponses et questions, p. 43 et 61.

43. Par "destin», il faut entendre le destin de l'être, c'est-à-dire la manifestation et le voilement de l'être qui forme la trame imprévisible et changeante de l'histoire. Sur la critique heideggerienne de la métaphysique de la subjectivité, cf. les cours sur Nietzsche (1936-1946) et le texte «Dépassement de la métaphysique».

44. La lecture heideggerienne de Jūnger aboutit en un séminaire en 1939-1940 (cf. le tome $\mathbf{9 0}$ de l'œuvre complète de Heidegger en allemand : Zu Ernst Jünger, publié par P. Trawny en 2004, p. 213-266). Notons par ailleurs que Heidegger et Jünger entretinrent une relation épistolaire dès les années 1930.

45. La mobilisation totale (trad. H. Plard et M. B. de Launay, Paris, Gallimard, coll. Tel, 1990), § 4, p. 115.

46. Ibid., p. 116.

47. "L'époque des "conceptions du monde"», p. 126, note 1.

48. La mobilisation totale, §6, p. 128.

49. “Dépassement de la métaphysique», § 26, p. 112.

50. Position avec laquelle le Jünger des années 1950, qui n'est plus l'essayiste "nouveau-nationaliste" et "réaliste héroïque» des années 1930, serait d'accord.

51. "Dépassement de la métaphysique», $\S 2$, p. 81-82; cf. § 3, p. 82-83. Ce passage fait référence au Travailleur de Jünger.

52. Cf. Heidegger, Réponses et questions, p. 41-42; et Jünger, La mobilisation totale.

53. La naissance de la tragédie, 1872 (éd. Colli-Montinari, trad. P. Lacoue-Labarthe, Paris, Gallimard, coll. Folio, 1986), § 119.

54. Lettre datée de février 1872, in : Querelle autour de La naissance de la tragédie (écrits et lettres de F. Nietzsche, F. Ritschl, E. Rohde, U. von WilamowitzMöllendorf et R. et C. Wagner, 1871-1873, trad. M. Cohen-Halimi, H. Poitevin et M. Marcuzzi, Paris, Vrin, 1995), p. 52.

55. La naissance de la tragédie, $\S 19$, p. 118.

56. Pour Nietzsche, dans les années 1870 , le développement d'une forme d'art complète et spécifiquement allemande est une étape nécessaire en vue d'accomplir la régénération de la civilisation allemande : ce sera la tragédie wagnérienne.

57. R. Wagner, "Lettre ouverte à Friedrich Nietzsche, professeur ordinaire de philologie classique à l'université de Bâle», 12 juin 1872 (in : Querelle autour de La naissance de la tragédie, op. cit.), p. 145.

58. Par-delà bien et mal. Prélude d'une philosophie de l'avenir (éd. Colli-Montinari, trad. C. Heim, Paris, Gallimard, coll. Folio, 1996), v, § 203.

59. Cf. Par-delà bien et mal, vi, § 208.

60. Par-delà bien et mal, III, $\S 61$. Nietzsche écrit plus loin : "comme tout le monde, je me suis mis à réfléchir sur des sujets qui ne me regardent en rien : premier symptôme d'infection politique» (VIII, § 251). 


\section{Martine Béland}

61. Pạr-delà bien et mal, III, § 61.

62. Cf. Par-delà bien et mal, III, $\S 61$.

63. Par-delà bien et mal, vı, $§ 211$.

64. Cf. Par-delà bien et mal, II, $\S 30$. Plus loin, Nietzsche affirme que la philosophie est foncièrement aristocratique : elle est le «lieu où l'on regarde les choses de haut en bas" (VI, § 205). Pour la référence à Schopenhauer sur l'aristocratie de la nature, cf. Contre la philosophie universitaire (trad. A. Dietrich, préface de M. Abensour et P.-J. Labarrière, Paris, Payot et Rivages, coll. Rivages poche, 1994), notamment aux pages 120,121 et 128 .

65. Sur la vie imprudente du philosophe, cf. Par-delà bien et mal, vI, $\S 205$, et sur le philosophe masqué : II, § 25 et 40 et IX, § 289.

66. "Le rectorat 1933-1934. Faits et réflexions" (in Heidegger, Écrits politiques 19331966, trad. F. Fédier, Paris, Gallimard, 1995), p. 219.

67. Nous précisons ici une idée suggérée par $M$. Abensour et P.-J. Labarrière (cf. Schopenhauer, Contre la philosophie universitaire, op. cit., p. 31).

68. E. Jünger, "Le Travailleur planétaire", entretien de 1981 (propos recueillis et traduits par F. de Towarnicki, in M. Haar (dir.), Cahier de l'Herne Martin Heidegger, Paris, L'Herne, 1983), p. 148.

69. Nous écrivons "le plus souvent possible», car il est révélateur de noter que les propos politiques les plus explicites et "complets" que nous avons du second Heidegger proviennent de l'entrevue de 1966, donc de réponses à des questions qui lui ont été posées, et face auxquelles Heidegger s'est souvent montré véritablement agacé (cf. p. 43 et 72 ) - des propos, qui plus est, dont le philosophe a interdit la divulgation et la publication avant sa mort (l'entrevue fut publiée en Allemagne en 1976). 